\title{
Consumo de heroína inyectada en Colombia y comportamientos de riesgo
}

\author{
Dedsy Berbesi, ${ }^{1}$ Angela Segura-Cardona, ${ }^{1}$ Liliana Montoya-Vélez, ${ }^{1}$ Pedro Mateu-Gelabert ${ }^{2}$
}

Artículo original

\section{SUMMARY}

Objective

To determine socio-demographic characteristics, prevalence of HIV risk, behaviors use.

\section{Methods}

We performed a cross-sectional study using sampling generated by the respondent; the sample consisted of 540 participants (Pereira: $n=300$; Medellín: $n=240$ ) over 18 years. The interview was conducted by an expert in counseling for HIV and sexually transmitted diseases. Ethical requirements were met. We used the software RDSAT.

\section{Results}

There are extensive networks of IDUs in both cities. The population of IDUs in both cities was characterized as mostly men between 18 and 24 , with secondary school, indicating very young populations. The data suggest a recent introduction of HIV into networks and a high degree of risk behavior for HIV spread in networks and used syringes received from others in the past six months.

\section{Conclusions}

The data in this report indicate a high risk for a possible expansion of HIV among injecting networks in Colombia supported the introduction of HIV into networks of injectors, high rates of injecting risk and a lack of injection as a practice of risk associated with HIV.

Key words: Heroin, sexual behavior, sampling studies, HIV.

\section{RESUMEN}

\section{Objetivo}

Determinar características socio-demográficas, prevalencia de $\mathrm{VIH}$ y comportamientos de riesgo en usuarios de drogas inyectables.

\section{Métodos}

Se realizó un estudio transversal, utilizando el muestreo generado por el entrevistado. Las muestras estuvieron compuestas por 540 participantes (Pereira: $n=300$; Medellín: $n=240$ ) mayores de 18 años. La entrevista la realizaba una persona experta en consejería para $\mathrm{VIH}$ y enfermedades de transmisión sexual. Se cumplieron los requerimientos éticos exigidos. Se utilizó la versión 6.01 del software RDSAT y la 8.0 de SPSS.

\section{Resultados}

En ambas ciudades existen amplias redes de usuarios de drogas inyectables. La población se caracterizó por ser en su mayoría hombres, entre 18 y 24 años, con estudios de secundaria. Los datos parecen indicar una reciente introducción de $\mathrm{VIH}$ en las redes y un alto grado de comportamiento de riesgo, incluyendo el uso de jeringas recibidas de otras personas en los últimos seis meses.

\section{Conclusiones}

Los datos indican una situación de alto riesgo para una posible expansión del VIH entre las redes de inyección en Colombia. Lo anterior se origina en la introducción del $\mathrm{VIH}$ en dichos grupos y un desconocimiento de la inyección como práctica de riesgo asociada con $\mathrm{VIH}$.

Palabras clave: Heroína, conducta sexual, muestreo, VIH.

\section{INTRODUCCIÓN}

La transmisión del VIH sigue siendo un importante reto para la salud pública mundial. ${ }^{1}$ En Colombia y el resto de Latinoamérica, las prácticas de inyección de heroína eran consideradas minoritarias. ${ }^{2}$ A partir de mediados de la década de 1990 se ha evidenciado un crecimiento constante y progresivo en la disponibilidad de heroína en el mercado nacional, y la subsecuente elevación de la prevalencia de consumo. ${ }^{3}$ Con un rápido incremento en la producción de heroína en el subcontinente, ${ }^{4}$ algunos investigadores han manifestado su preocupación por el consumo de drogas inyectables en Colombia y la necesidad de contención de una posible epidemia de VIH..$^{5-7}$

El uso de drogas intravenosas ha sido un factor de riesgo muy importante desde el inicio de la epidemia del sida, pero en los últimos años ha cobrado más importancia. Alrededor de 20 países han tenido reportes de prevalencias mayores a $20 \%$ en usuarios de drogas inyectables (UDI) entre los cuales se encuentran Rusia, Ucrania, Italia, Países Bajos, Portugal, España, India, China, Argentina, Brasil, Uruguay, Puerto Rico, Estados Unidos y Canadá. ${ }^{8}$

Facultad de Medicina. Universidad CES. Medellín, Colombia.

2 Investigador de National Development Research Institutes. New York, USA

Correspondencia: Dedsy Berbesí Fernández, Calle 10 A, 22-04. Medellín, Colombia. Tel. (+054) 444-0555. Fax: 268-2876. E-mail: dberbesi@ces.edu.co

Recibido: 14 de marzo de 2012. Aceptado: 2 de agosto de 2012. 
La epidemia por el VIH entre los UDI se diferencia de las de otras poblaciones porque pueden propagarse rápidamente dentro de esta comunidad y de ahí pasar a la población en general. ${ }^{9}$ Dentro de los UDI, el principal factor de riesgo que aumenta la posibilidad de transmisión es el intercambio de jeringas y agujas, principalmente en personas jóvenes, donde se encuentran informes de infección por esta vía de hasta $43.5 \% .{ }^{10}$ Si bien los más afectados son los hombres, algunos estudios informan que $31 \%$ de las mujeres aceptaron haberse inyectado con agujas previamente usadas, ${ }^{11}$ lo que las incluye también en el grupo de riesgo. Además, las mujeres que son parejas sexuales de un UDI tienen mayor tendencia a estar comprometidas como receptoras después de la inyección de su compañero sexual; esto es particularmente común en países en vías de desarrollo. ${ }^{12}$

Por sus prácticas sexuales, los UDI tienen mayor riesgo de adquirir enfermedades de transmisión sexual y en especial VIH. Las mujeres también presentan mayor tendencia a encontrarse inmersas en conductas sexuales riesgosas como intercambiar sexo por dinero o drogas, tener múltiples parejas sexuales, compartir agujas durante la relación sexual o tener sexo no protegido con una pareja UDI. ${ }^{13}$

Algunos factores socio-culturales que influyen en el mayor riesgo de la adquisición del $\mathrm{VIH}^{14}$ incluyen la baja capacidad para tomar decisiones, la falta de acceso a la educación, los problemas familiares y sociales que generen comportamientos psicopáticos que se agravan con la desocupación laboral y dificultades económicas graves que llevan a la persona a vivir un estado de inequidad social, lo que puede influir en la mayor transmisión de VIH en esta población. ${ }^{15}$

Para determinar el consumo de drogas inyectables en Colombia, se eligieron dos ciudades, Medellín y Pereira, situadas en las zonas de cultivo de amapola (eje cafetalero). Utilizando el método de muestreo guiado por el entrevistado ${ }^{16}$ (RDS por sus siglas en inglés), ${ }^{17,18}$ se evaluaron prácticas de riesgo y prevalencia del VIH entre los usuarios de drogas inyectables en estas dos ciudades. El muestreo basado en el entrevistado ha surgido como una alternativa para poblaciones ocultas. ${ }^{19,20}$

El objetivo de esta investigación fue identificar los comportamientos de riesgo para la transmisión del VIH en los usuarios de drogas inyectables de dos ciudades de Colombia: Medellín-Antioquia y Pereira-Risaralda.

\section{MATERIAL Y MÉTODOS}

La recolección de información se llevó a cabo durante el segundo semestre de 2010 en Medellín y Pereira. Las muestras estuvieron compuestas por un total de 540 participantes (Pereira: n=300; Medellín: n=240) mayores de 18 años. Estas ciudades fueron seleccionadas por dos razones principales: en primer lugar, ambas tenían estudios previos ${ }^{21,22}$ que informaban de un número importante de usuarios de drogas inyectables; en segundo lugar, estas ciudades han sido epi- centros del microtráfico. ${ }^{23}$ Estas dos ciudades proporcionaron una muestra diversa de personas usuarias de drogas inyectables de Colombia y una comparación de dos diferentes tendencias epidemiológicas en zona urbana.

Debido al tipo de población estudiada, no fue posible contar con un marco muestral conocido que permitiera diseñar una muestra probabilística convencional, por lo que se optó por una muestra de referencia en cadena basada en quienes se entrevista, método que ha comprobado su utilidad para acceder a poblaciones de difícil acceso, como son los usuarios de drogas inyectables, y que consiste en un muestreo con referencia en cadena, pero que incorpora teorías de redes sociales para obtener una muestra que se aproxime a la población estudiada.

El reclutamiento se inicia con un grupo de "semillas" o participantes no aleatorios de la población objetivo. En el caso de la presente investigación se determinó una cantidad inicial de tres semillas, de las posibles para cada ciudad. Desde estas semillas se iniciaron las cadenas de reclutamiento, con lo que se logró una muestra regional de mínimo 200 participantes. A estas personas se les entregaron dos tipos de incentivo, un incentivo primario o por participar en el estudio y otro secundario, vinculado al reclutamiento exitoso de tres nuevos participantes (los valores variaron entre aproximadamente 4 y 5 dólares estadounidenses, respectivamente).

La selección de las semillas se basó en cuatro variables sociodemográficas, que se consideró que podían tender a conformar redes y subgrupos dentro de los UDI. Se identificaron 20 semillas potenciales (Pereira: $n=12$; Medellín: $n=8$ ), que variaron según el estrato socioeconómico, el género, el nivel educativo (primaria, bachillerato y universitario) y la edad. Las semillas fueron reclutadas a través de informantes claves.

Para iniciar la segunda etapa, se explicó a las semillas entrevistadas el proceso de reclutamiento, que incluía como criterios de inclusión el tener 18 años de edad o más y ser consumidor en cada ciudad de drogas por vía inyectable. Los compañeros, a su vez, reclutaron a otros compañeros en el estudio. El tamaño de la muestra objetivo se alcanzó en un plazo de ocho semanas.

La entrevista la realizaba una persona experta en consejería para VIH y enfermedades de transmisión sexual, y tenía una duración promedio de 60 minutos. El instrumento incluyó preguntas sobre características demográficas, conocimientos sobre VIH y una prueba de VIH. Se cumplió con los requerimientos éticos requeridos y aprobados. Para estimar la prevalencia de las variables de interés, se utilizó la versión 6.01 del software RDSAT.

\section{RESULTADOS}

\section{Configuración de las redes}

En cada ciudad se lograron tres redes a partir de tres semillas como se puede observar en la figura 1. 


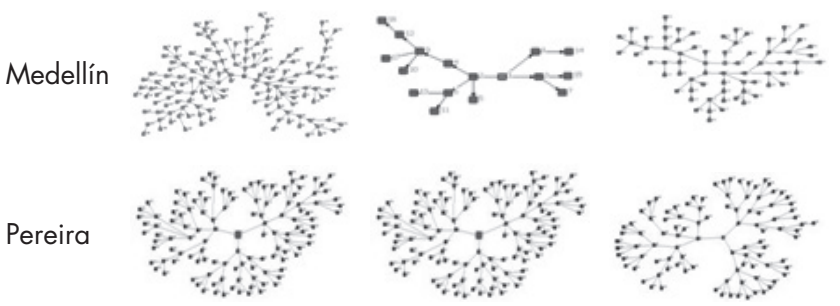

Figura 1. Redes sociales en Medellín y Pereira. Prevalencia de VIH en usuarios de drogas inyectables. 2011

\section{Características demográficas}

La población UDI se caracterizó por ser: hombres (92.8\%), solteros $(81.3 \%)$, menores de 30 años $(85.7 \%)$, con estudios de secundaria (89.5\%). El rango de edad era de 18 a 58 (25 \pm 6.3$)$. El $76.7 \%$ tenía un estrato socioeconómico bajo, un $20 \%$ estrato medio y un $3 \%$ estrato alto.

\section{Uso de drogas y alcohol}

La mayoría de los participantes en el estudio eran consumidores de drogas recientes, con un tiempo medio de 4 $( \pm 3)$ años. Las drogas usadas actualmente incluían basuco $(40.7 \%)$, marihuana $(88 \%)$ y cocaína $(60 \%)$. El abuso de alcohol fue reportado por $67 \%$. Todos eran usuarios de heroína inyectable.

\section{Comportamientos sexuales}

El número actual de parejas sexuales variaba entre los grupos. Más de la mitad de los participantes (54\%) informaron tener relaciones sexuales con parejas ocasionales durante los últimos seis meses, un $27 \%$ reportaron tener entre 2 y 10 parejas sexuales ocasionales y un $4 \%$ reportaron tener más de 10 parejas sexuales en los últimos seis meses.

El 52\% refirió tener sexo con parejas casuales y el 15\% con clientes; el $65 \%$ de los participantes tenía sexo con una pareja sexual permanente. El 10\% informó de relaciones sexuales con múltiples parejas por drogas y/o por dinero, siendo para las mujeres cuatro veces la probabilidad de intercambiar sexo por drogas y/o dinero que los hombres $(\mathrm{OR}=4.71$; IC 95\% 2.2-10.7, $\mathrm{p}=0.00)$.

El uso constante de condón fue indicado sólo por $16 \%$ de los participantes y $14 \%$ de los hombres lo usó durante el coito vaginal. Aquellos que tenían más parejas refirieron un uso similar del condón durante el sexo vaginal (16\%) y un 33\% de la población del estudio afirmó que ellos nunca habían usado un condón.

Las personas que comparten agujas tienen dos veces el riesgo de no utilizar condón ( $\mathrm{OR}=2.10$; IC 95\% 1.2-3.5; $\mathrm{p}=0.00)$. El 27\% de los UDI que comparten agujas tienen relaciones sexuales con otras personas que también son UDI.

El $44 \%$ de los UDI ha usado en los últimos seis meses jeringas recibidas de otras personas. La limpieza de dichos equipos la hacen generalmente con agua $(67 \%)$, seguida de alcohol $(8.5 \%)$ o con agua hirviente $(2.4 \%)$. No se encontraron diferencias por sexo al analizar el hábito de compartir jeringas, pero sí con respecto al vivir solo, consumir basuco (que puede disminuir el efecto de la heroína), ruedas o éxtasis (cuadros 1 y 2).

\section{Prevalencia de VIH}

La prevalencia de VIH estimada para la población de UDI fue de $2.0 \%$ [IC 95\%: 0.7\%-3.3\%]; para los hombres fue de 1.99\% [IC 95\%: 0.67\%-3.3\%] y para las mujeres de $2.56 \%$ [IC 95\%: $0.06 \%-13.4 \%$ ], sin diferencias estadísticamente significativas por sexo.

\section{DISCUSIÓN}

Se estima que $29 \%$ de los más de dos millones de latinoamericanos usuarios de drogas inyectables está infectado por el VIH. ${ }^{24}$ En la presente investigación se identificó la presencia de VIH en población usuaria de drogas inyectables en dos ciudades de Colombia y la existencia de un consumo esta-

Cuadro 1. Características sociodemográficas de los UDI

\begin{tabular}{|c|c|c|c|c|c|c|c|c|}
\hline \multirow[b]{3}{*}{ Variable } & \multicolumn{4}{|c|}{ Comparten jeringas } & \multirow{3}{*}{$\begin{array}{c}\text { Chi } \\
\text { cuadrada }\end{array}$} & \multirow{3}{*}{$\begin{array}{l}\text { Valor } \\
\text { de } p\end{array}$} & \multirow[b]{3}{*}{ OR } & \multirow[b]{3}{*}{ IC (95\%) } \\
\hline & \multicolumn{2}{|c|}{ Sí } & \multicolumn{2}{|c|}{ No } & & & & \\
\hline & $\mathrm{N}$ & $\%$ & $\mathrm{n}$ & $\%$ & & & & \\
\hline $\begin{array}{l}\text { Sexo } \\
\text {-Hombres } \\
\text {-Mujeres }\end{array}$ & $\begin{array}{r}213 \\
21\end{array}$ & $\begin{array}{r}91.0 \\
9.0\end{array}$ & $\begin{array}{r}288 \\
18\end{array}$ & $\begin{array}{r}94 \\
6\end{array}$ & 1.89 & 0.111 & 0.63 & $(0.32-1.21)$ \\
\hline $\begin{array}{l}\text { Vive con su } \\
\text { - No } \\
\text { - Sí }\end{array}$ & $\begin{array}{r}\text { adres } \\
139 \\
95\end{array}$ & $\begin{array}{l}59.4 \\
40.6\end{array}$ & $\begin{array}{l}156 \\
150\end{array}$ & $\begin{array}{l}51 \\
49\end{array}$ & 3.79 & 0.031 & 1.4 & $(0.9-1.9)$ \\
\hline $\begin{array}{l}\text { Vive solo } \\
\text { - Sí } \\
\text { - No }\end{array}$ & $\begin{array}{r}73 \\
161\end{array}$ & $\begin{array}{l}31.2 \\
68.8\end{array}$ & $\begin{array}{r}67 \\
239\end{array}$ & $\begin{array}{l}22 \\
78\end{array}$ & 5.97 & 0.01 & 1.67 & $(1.0-2.3)^{*}$ \\
\hline
\end{tabular}

* Valor de $p<0.05$ e intervalo de confianza con límite inferior mayor a 1. 
Berbesi et al.

Cuadro 2. Consumo de otras drogas en la población UDI

\begin{tabular}{|c|c|c|c|c|c|c|c|c|}
\hline \multirow[b]{3}{*}{ Variable } & \multicolumn{4}{|c|}{ Comparten jeringas } & \multirow{3}{*}{$\begin{array}{c}\text { Chi } \\
\text { cuadrada }\end{array}$} & \multirow{3}{*}{$\begin{array}{l}\text { Valor } \\
\text { de } p\end{array}$} & \multirow[b]{3}{*}{ OR } & \multirow[b]{3}{*}{ IC (95\%) } \\
\hline & \multicolumn{2}{|c|}{ Sí } & \multicolumn{2}{|c|}{ No } & & & & \\
\hline & $\mathrm{N}$ & $\%$ & $\mathrm{n}$ & $\%$ & & & & \\
\hline \multicolumn{9}{|l|}{ Basuco } \\
\hline - Sí & 110 & 47.0 & 110 & 36 & 6.72 & 0.006 & 1.58 & $(1.11-2.23)^{*}$ \\
\hline - No & 124 & 53.0 & 196 & 64 & Inhalables & & & \\
\hline - Sí & 53 & 22.6 & 49 & 16 & 3.81 & 0.033 & 1.53 & $(0.99-2.36)$ \\
\hline - No & 181 & 77.4 & 257 & 84 & & & & \\
\hline \multicolumn{9}{|l|}{ Ruedas } \\
\hline - Sí & 161 & 68.8 & 163 & 53 & 13.34 & 0.000 & 1.93 & $(1.35-2.76)$ * \\
\hline $\begin{array}{l}\bullet \text { No } \\
\text { Éxtasis }\end{array}$ & 73 & 31.2 & \multicolumn{5}{|c|}{ Éxtasis } & \\
\hline - Sí & 33 & 14.1 & 26 & 8 & 4.28 & 0.027 & 1.76 & $(1.02-3.04)^{*}$ \\
\hline $\begin{array}{l}\text {-No } \\
\text { Cigarrillo }\end{array}$ & 201 & 85.9 & 280 & 92 & & & & \\
\hline - Sí & 217 & 92.7 & 270 & 88 & 3.03 & 0.0816 & 1.70 & $(0.93-3.11)$ \\
\hline $\begin{array}{l}\text {-No } \\
\text { Bebida }\end{array}$ & 17 & 7.3 & 36 & 12 & & & & \\
\hline • Sí & 167 & 71.4 & 194 & 63 & 3.8 & 0.031 & 1.43 & (0.99-20.7) \\
\hline • No & 67 & 28.6 & 112 & 37 & & & & \\
\hline Total & 234 & & 306 & & & & & \\
\hline
\end{tabular}

* Valor de $p<0.05$ e intervalo de confianza con límite inferior mayor a 1.

blecido de heroína vía parenteral con conductas de riesgo, lo que los convierte en factores predisponentes para una posible expansión de VIH entre los UDI y sus redes sexuales como se ha evidenciado en otras investigaciones. ${ }^{25,26}$

Los datos de este informe indican una situación de alto riesgo para una rápida expansión del VIH entre las redes de inyección por el desconocimiento de medidas efectivas para la limpieza de equipos que son reusados y/o compartidos. Si se aplican los conocimientos científicos y la experiencia de las últimas décadas en la prevención de epidemias en usuarios de drogas inyectables, se tendría una oportunidad única para prevenir la rápida expansión de VIH en la redes de UDI. La voluntad por parte de los UDI de poner en práctica medidas preventivas referidas por los entrevistados debe ser reforzada por medio de programas que proporcionan conocimiento sobre prácticas efectivas en la prevención de VIH. ${ }^{27}$ Cabe anotar que la mayoría utiliza el agua para realizar la limpieza del equipo de inyección, lo que revela un desconocimiento sobre medidas efectivas de desinfección.

La tendencia de los UDIs de tener más de un comportamiento de alto riesgo, como el comercio del sexo, multiplica la probabilidad de infección. La evidencia indica que la transmisión sexual tanto entre inyección como no inyección de consumidores de droga desempeña un papel importante en la propagación del VIH a nivel mundial. ${ }^{28}$ Así, los consumidores de droga, a pesar de su conducta de inyectar droga, funcionan como una población puente y facilitan la propagación de enfermedades transmitidas por sangre más allá de la población que consume drogas. ${ }^{29}$

La disponibilidad y accesibilidad a la heroína en Colombia y su impacto en la transmisión del virus de la inmu- nodeficiencia humana por vía sexual o inyectable impone el diseño de estrategias orientadas a controlar una inminente epidemia, por lo que se requiere la aplicación de experiencias y medidas eficaces para reducir riesgos de infección. ${ }^{30}$ Se requiere incrementar el grado de conocimiento sobre los riesgos de VIH y otras infecciones (hepatitis $\mathrm{C}$, infecciones bacterianas) relacionadas con las prácticas de inyección. ${ }^{31}$

En algunos países ya se están implementando estrategias para la disminución de la transmisión de esta patología; algunas de ellas son: terapia de sustitución de opio, consejería y programas de intercambio de jeringas, eliminación segura de jeringas y agujas contaminadas.

Es muy importante que los gobiernos continúen invirtiendo en políticas de salud pública y en estrategias de prevención de VIH para los UDI, y focalizar los esfuerzos en las poblaciones de riesgo, ya que en varios países latinoamericanos, incluida Colombia, son los grupos donde se concentra esta infección.

\section{REFERENCIAS}

1. OMS, UNODC. Informe hacia el acceso universal; 2010. Disponible en http://www.who.int/hiv/universalaccess2010/es/index.html.Acceso 17 de junio de 2011.

2. Organización Panamericana de la Salud. Oficina de Naciones Unidas. VIH y SIDA en las Américas España: 2001.

3. Mejía Motta I E. La inyección de drogas en Bogotá: Una realidad oculta. Bogotá, Colombia; Presidencia de la Republica de Colombia; 2003.

4. Naciones Unidas. Oficina contra la Droga y el Delito. Tendencias mundiales de las drogas ilícitas. Disponible en: http://www.portalfarma.com/pfarma/taxonomia/general/gp000012.nsf/voDocumentos/1A6BB7A9C264ABA4C1256F89005D0099/\$File/43-49-Tendencias.pdf. Acceso el 17 de junio de 2011. 
5. Mejia IE, Perez A. La inyección de drogas en Bogotá: una amenaza creciente. Adicciones 2005;17 (3):251-260.

6. Usuarios de drogas inyectables y acceso a tratamiento. Disponible en: http://www.icw.org/files/IDUES.pdf. Acceso el 14 de enero de 2011.

7. Zurmühl U, Lechner I. VIH/SIDA en Latinoamérica y el Caribe. FAKT. Alemania.2006. Disponible en: http://www.portalsida.org/repos/vihsida_en_el_latinoamerica_y_el_caribe.pdf. Acceso el 17 de junio de 2011.

8. Aceijas C, Stimson GV, Hickman M, Rhodes T. Global overview of injecting drug use and HIV infection among injecting drug users. AIDS 2004;18(17):2295-2303.

9. The science of improving lives. Usuarios de drogas inyectables. Disponible en: http://www.fhi.org/sp/Topics/Injection+Drug+Users+topic+page.htm.

10. Chu TX, Levy JA. Injection drug use and HIV/AIDS transmission in China. Cell Res 2005;15(11-12):865-869.

11. Reid SR. Injection drug use, unsafe medical injections, and HIV in Africa: a systematic review. Harm Reduct J 2009;6:24.

12. Ross MW, McCurdy SA, Kilonzo GP, Williams ML et al .Drug use careers and blood-borne pathogen risk behavior in male and female Tanzanian heroin injectors. Am J Trop Med Hyg 2008;79(3):338-343.

13. Kozlov AP, Shaboltas AV, Toussova OV, Verevochkin SV et al. HIV incidence and factors associated with HIV acquisition among injection drug users in St Petersburg, Russia. AIDS 2006;20(6):901-906.

14. Mathers BM, Degenhardt L, Ali H, Wiessing L e $t$ al. HIV prevention, treatment, and care services for people who inject drugs: a systematic review of global, regional, and national coverage. Lancet 2010;375(9719):1014-1028.

15. Steffanie A, Strathdee TB. HIV and risk environment for injecting drug users: the past, present and future. Lancet 2010;376:268-284.

16. Medina-Mora ME. Estudio de poblaciones ocultas y de difícil acceso. Disponible en: http://www.conadic.salud.gob.mx/pdfs/publicaciones/ obs02met.pdf

17. Heckathorn DD. Respondent-driven sampling: a new approach to the study of hidden populations. Social Problems 1997; 44:174-199.

18. Heckathorn DD, Semaan S, Broadhead R, Hughes JJ. Extensions of respondent-driven sampling: A new approach to the study of injection drug users aged 18-25. AIDS Behavior 2002; 6:55-67.

19. UNAIDS. Guidelines on estimating the size of populations most at risk to HIV. Disponible http://www.unaids.org/en/media/unaids/contentassets/restore/2011. Estimating_Populations_en.pdf. Acceso el 17 de junio de 2011.
20. ONUSIDA. Marco para la vigilancia y la evaluación de los programas de prevención del VIH dirigidos a las poblaciones de mayor riesgo. Suiza; 2008.

21. Isaza C, Suarez P, Henao J, Gonzalez M. Características demográficas y de consumo en pacientes adictos a heroína o derivados de la coca. Investigaciones Andina 2010; 12:24-34.

22. Castaño G, Calderón GA. Consumo de heroína en Colombia, prácticas relacionadas e incidencia en la salud pública. Revista Cubana Salud Pública 2010; 36(4):311-322.

23. Perez A. Micro tráfico de heroína, un tercer estudio realizado en Medellín y Bogotá en el 2009. Colombia: Ministerio de Protección Social; 2010.

24. ONUSIDA. Situación de la Epidemia de SIDA. Disponible en http:// www.unaids.org/globalreport/Global_report_es-hts. Acceso el 17 de junio de 2011.

25. Metzger DS, Navaline H. Human immunodeficiency virus prevention and the potential of drug abuse treatment. Clinical Infectious Diseases 2003;5(37 Supl 5):S451-S456.

26. World Health Organization. Evidence for action: Effectiveness of drug dependence treatment in preventing HIV among injection drug users. Ginebra, Suiza: 2005.

27. Center for Disease Control and Prevention. Management of Possible Sexual, Injecting-Drug-Use, or Other Nonoccupational Exposure to HIV, Including Considerations Related to Antiretroviral Therapy Public Health Service Statement Morbidity and Mortality Weekly Report 1998 Sept 25;47(RR17):1-14. Disponible en: http://www.cdc.gov/mmwr/ preview/mmwrhtmil/00054952.htm. Accedo el 17 de junio de 2011.

28. Ross MW, Williams ML. Sexual behavior and illicit drug use. Annu Rev Sex Res 2001;12:290-310.

29. Shehane E, Jacobson JO, Iguchi MY. Un estudio para identificar comportamientos de riesgo y necesidades de prevención de VIH/SIDA en Centros de Tratamiento de Drogadicción en la Ciudad de Guatemala y San Salvador. Guatemala, 2008. Disponible en http://www.seguridad. gob.sv/observatorio/demanda/2008/VIH:UDI_Esp_Final.pdf. Acceso el 17 de junio de 2011.

30. Hacker MA, Malta M, Enriquez M, Bastos F. Human immunodeficiency virus, AIDS, and drug consumption in South America and the Caribbean: epidemiological evidence and initiatives to curb the epidemic. Rev Panam Salud Publica 2005;18(4-5):303-13.

31. Rodríguez CM, Marques LF, Touzé G. HIV and injection drug use in Latin America. AIDS 2002;16 (Supl 3):S34-S41.

Artículo sin conflicto de intereses 